\title{
Newborn infant parasympathetic evaluation for the assessment of analgosedation adequacy in infants treated by mechanical ventilation - a multicenter pilot study
}

\author{
Wojciech Walas ${ }^{1}$, Ewelina Malinowska², Zenon P. Halaba ${ }^{3}$, Tomasz Szczapa ${ }^{4}$, Julita Latka-Grot $^{5}$, \\ Magdalena Rutkowska ${ }^{6}$, Agata Kubiaczyk ${ }^{4}$, Monika Wrońska ${ }^{7}$, Andrzej Piotrowski ${ }^{7}$, \\ Michał Skrzypek ${ }^{8}$, Mickael Jean-Noel ${ }^{9}$, Iwona Maroszyńska²
}

\author{
'Department of Pediatric Anesthesiology and Intensive Care, Institute of Medical \\ Sciences, University of Opole, Opole, Poland \\ 2Department of Intensive Care and Congenital Malformations of Newborns and \\ Infants, Polish Mother's Memorial Hospital Research Institute, Lodz, Poland \\ ${ }^{3}$ Department of Pediatrics, Institute of Medical Sciences, University of Opole, Opole, Poland \\ ${ }^{4}$ Department of Neonatology, Neonatal Biophysical Monitoring and Cardiopulmonary \\ Therapies Research Unit, Poznan University of Medical Sciences, Poznan, Poland \\ ${ }^{5}$ Neonatal Department, Children's Memorial Health Institute, Warsaw, Poland \\ ${ }^{6}$ Department of Neonatology, Institute of Mother and Child, Warsaw, Poland \\ ${ }^{7}$ Department of Anaesthesiology and Intensive Care, Children's Memorial Health \\ Institute, Warsaw, Poland \\ ${ }^{8}$ Department of Biostatistics, School of Public Health in Bytom, Medical University \\ of Silesia in Katowice, Bytom, Poland \\ ${ }^{9}$ INSERM CIC-IT 1403, Lille University Hospital, Lille, France
}

Submitted: 30 October 2020; Accepted: 12 March 2021;

Online publication: 24 March 2021

Arch Med Sci 2021; 17 (6): 1651-1656

DOI: https://doi.org/10.5114/aoms/134234

Copyright (c) 2021 Termedia \& Banach

\section{Abstract}

Introduction: Adequate analgosedation is important in infants treated in pediatric/neonatal intensive care units (P/NICUs), because both too deep and insufficient analgosedation is disadvantageous. To assess the severity of pain, several behavioral and behavioral-physiological scales are used, but their usefulness is limited. It is therefore justified to search for additional methods to assess the adequacy of analgosedation in these patients. The aim of the present study is to evaluate the usefulness of Newborn Infant Parasympathetic Evaluation (NIPE) in the assessment of analgosedation quality in infants requiring mechanical ventilation, who are treated in P/NICUs.

Material and methods: We performed simultaneously 180 COMFORT-B assessments and heart rate variability measurements using a NIPE monitor in 30 mechanically ventilated infants receiving analgosedation. A generalized linear mixed model with the logit link function was used in order to perform logistic regression analysis to assess the relationship between NIPEi/NIPEm and deep sedation.

Results: The multivariable logistic regression model showed that NIPEi and NIPEm values were higher when analgosedation was deep as compared to when it was moderate or insufficient (OR $(95 \% \mathrm{CI})$ : NIPEm - 1.065 (1.0071.126), $p=0.03$; NIPEi - 1.068 (1.016-1.123), $p=0.01)$.

Conclusions: The NIPE indexes are significantly higher in patients whose assessment on the behavioral scale indicates deep analgosedation as compared to those in whom it indicates moderate or insufficient analgosedation. Allowing continuous monitoring, the NIPE device may be a valuable assisting tool in the assessment of analgosedation quality in mechanically ventilated newborns and infants.

Key words: infant, pain, analgosedation, heart rate variability, Newborn Infant Parasympathetic Evaluation, COMFORT-B scale.
Corresponding author: Prof. Zenon P. Halaba Department of Pediatrics Institute of Medical Sciences University of Opole 48 Oleska St 45-052 Opole, Poland E-mail: zhalaba@uni.opole.pl 


\section{Introduction}

Pain relief is one of the basic elements in the treatment of newborns and children in the intensive care unit. Inadequate pain management is a cause of suffering. It may also cause complications and, moreover, in neonates and infants can produce long-term consequences, especially regarding the anatomical and functional development of the brain [1-10]. On the other hand, overuse of painkillers can also cause side effects [11-13]. The selection of appropriate hypnotics and painkillers as well as their dosage must be individually tailored and should take into account the type of disease and the type of medical procedures applied. In addition, the need for these drugs may vary from patient to patient [14-18]. This requires an appropriate assessment of the severity of pain, which should be perceived, monitored and documented as the "fifth vital sign". The literature shows that many newborns and infants treated in intensive care units experience pain [14-19]. In these patients, as in intubated and mechanically ventilated adults, the assessment of pain intensity is difficult due to the lack of verbal contact, and pain monitoring is performed using behavioral and behavioral-physiological scales based on the assessment of various behavioral elements and basic vital signs. These scales serve as the only validated method of pain assessment in newborns and infants so far, but they have many limitations [19-23]. Their use is time-consuming, requires experience, and the evaluation is not continuous, which may result in missing pain incidents. In addition, different scales are designed for different patients depending on maturity and clinical situation, which in practice makes the interpretation of the results difficult. In neonates and infants receiving analgosedation, pain assessment is essential, as these patients usually undergo invasive procedures and are often subject to post-operative care. Furthermore, in these patients use of behavioral scales may be unreliable because administration of anesthetic drugs and hypnotic agents affects the assessed parameters. Therefore, the search for instrumental methods supporting the evaluation of pain intensity in these patients is of particular importance. One of these is the assessment of the balance between the sympathetic and parasympathetic systems based on the analysis of heart rate variability. The aim of the study is to evaluate the usefulness of the Newborn Infant Parasympathetic Evaluation (NIPE) monitor (MdolorisMedical Systems, Loos, France) for pain assessment in newborns and infants treated in the intensive care unit, based on the comparison of the NIPE index value and simultaneous assessment using a behavioral (COMFORT-B) scale.
This was a prospective, observational study which did not require any changes in regard to the standard treatment of patients included. The acceptance of the local Ethical Committee was granted (270: 11.10.2018).

\section{Material and methods}

\section{Patients}

We enrolled 30 neonates and infants: 17 (57\%) males and 13 (43\%) females treated in pediatric/ neonatal intensive care units (P/NICUs) due to respiratory failure caused by respiratory distress syndrome (RDS) or pneumonia. Inclusion criteria were as follows: gestational age at birth over 26 weeks and chronological age less than 1 year, mechanical ventilation through an endotracheal tube, analgosedation (fentanyl: $1-2 \mu \mathrm{g} / \mathrm{kg} / \mathrm{h}$, midazolam: $0.1-0.2 \mathrm{mg} / \mathrm{kg} / \mathrm{h}$ ). Exclusion criteria included bradycardia < 80 beats per minute (bpm), tachycardia > 250 bpm, any cardiac rhythm other than sinus one, use of medications that have effects on sympathetic and parasympathetic activity (e.g. $\beta$-blockers) during a period of 7 days before the study, intraventricular hemorrhage of grade IV, severe perinatal asphyxia treated by therapeutic hypothermia, central nervous system congenital malformations, seizures within 7 days preceding the study, neuromuscular diseases and analgosedation other than fentanyl/midazolam. Legal guardians of all subjects were given informed consent forms and agreed to participation in the study.

\section{Protocol}

Fentanyl and midazolam were administered by continuous infusion in accordance with the treatment schemes of the participating departments and the study did not affect it. Patients were constantly monitored using a NIPE monitor. They were also periodically evaluated using the COMFORT-B scale. The intervals between the COMFORT-B scorings were at least $1 \mathrm{~h}$ long. Patients were not disturbed by any additional procedures during the 30-minute period before this assessment.

\section{Pain assessment}

The COMFORT-B scale is designed for mechanically ventilated patients and is used to assess the degree of analgosedation [24]. The scale consists of six behavioral items: alertness, calmness, respiratory response, body movements, facial tension and muscle tone. Each variable may be assigned a score from 1 (the highest sedation level) to 5 (the lowest sedation level). The minimum and maximum total score is 6 and 30 respectively. Scores 6-10 indicate deep analgosedation, scores 11-23 
indicate that the patient is moderately sedated, and scores $>23$ indicate insufficient sedation. The COMFORT-B assessment was performed by experienced neonatologists who did not have access to the measurements provided by the NIPE monitor.

Newborn Infant Parasympathetic Evaluation is a monitor used to measure the intensity of pain in patients under 2 years of age. Heart rate variability (HRV) analysis is based on the recording obtained from a standard patient cardiac monitor via analog output, followed by real-time filtering and identification of the high-frequency component of HRV using fast wavelet transform filtering. The NIPE monitor displays two values of the NIPE index continuously: the NIPEm is computed as a mean value over 20 min, whereas the instantaneous NIPEi provides information regarding shortterm HRV-analysis. This monitor presents values from 0 to 100 points; the stronger the pain, the lower is the result displayed. A comprehensive description of these methodologies has been published by Jeanne et al., Faye et al. and De Jonckheere et al. [25-27].

\section{Statistical analysis}

The statistical analysis was performed using SAS software version 9.4 (SAS Institute Inc., Cary, North Carolina, USA) and R version 3.5.1 (The R Foundation for Statistical Computing). Descriptive statistics were expressed as median and the lower and the upper quartile. Due to multiple measures per patient, we used a generalized linear mixed model with the logit link function to perform logistic regression analysis in order to assess the relationship between NIPEi/NIPEm and deep sedation. We began with univariable logistic regression models, then to adjust for gender, postmenstrual age (PMA), and weight during assessment, we calculated the multivariable logistic regression model. Only statistically significant variables were left in the final model. Because of the strong correlation between NIPEi and NIPEm we decided to compute two separate models for these variables. Results were presented as odds ratios (OR) with 95\% confidence intervals $(95 \% \mathrm{Cl})$. We assumed the significance level $p<0.05$.
Table I. Characteristics of the studied group $(n=30)$

\begin{tabular}{|c|c|}
\hline Parameter & Value \\
\hline Male & $17(57)$ \\
\hline Female & $13(43)$ \\
\hline Vaginal delivery & $12(40)$ \\
\hline Caesarean section & $18(60)$ \\
\hline Gestational age [weeks] & $37(33-38)$ \\
\hline Birth weight $[\mathrm{g}]$ & $2775(1840-3500)$ \\
\hline Age at the time of the study [days] & $14(4-36)$ \\
\hline $\begin{array}{l}\text { Postmenstrual age at the time } \\
\text { of the study [weeks] }\end{array}$ & $41(36-42)$ \\
\hline Weight at the time of the study [g] & $3200(2410-3800)$ \\
\hline
\end{tabular}

\section{Results}

Thirty patients were included, in whom an assessment using the COMFORT-B scale was performed 200 times. Twenty NIPE recordings (10\% of all) were illegible for assessment due to artifacts and were excluded. In the final analysis, 180 simultaneous assessments of the COMFORT-B score and NIPE level in 30 patients were analyzed. The characteristics of the study group are shown in Table I.

We distinguished two subgroups depending on the COMFORT-B score: deep analgosedation (COMFORT-B $\leq 10)$ was observed in 117-measurements (65\%), and moderate/insufficient analgosedation (COMFORT-B > 10) was observed in 63 measurements (35\%). Although medians in both subgroups varied only a little, the differences were statistically significant. When comparing obtained NIPE results in these two subgroups, the multivariable logistic regression model showed that NIPEi and NIPEm values were higher in the deep analgosedation subgroup and the increase in NIPEi and NIPEm values by 1 unit raises the odds of deep analgosedation approximately by $7 \%$. Furthermore, we found that lower postmenstrual age is also associated with excessive sedation. Gender, type of delivery, birth weight as well as gestational age had no influence on the sedation level. Our results are presented in Table II and in Figure 1.

Table II. Newborn Infant Parasympathetic Evaluation (NIPE) indexes as well as postmenstrual age (PMA) and weight measurements during assessment $(N=180)$ with univariable logistic regression odds ratios

\begin{tabular}{|c|c|c|c|c|}
\hline \multirow[t]{2}{*}{ Variable } & $\begin{array}{c}\text { Deep } \\
\text { analgosedation }\end{array}$ & $\begin{array}{c}\text { Moderate/insufficient } \\
\text { analgosedation }\end{array}$ & \multirow[t]{2}{*}{ OR $(95 \% \mathrm{Cl})$} & \multirow[t]{2}{*}{$P$-value } \\
\hline & \multicolumn{2}{|c|}{ Median (lower - upper quartile) } & & \\
\hline PMA [weeks] & $43(38-60)$ & $76(52-97)$ & $0.932(0.896-0.969)^{a}$ & 0.0005 \\
\hline Weight [g] & $3100(1935-3600)$ & $3660(3200-4340)$ & $0.824(0.727-0.935)^{b}$ & 0.003 \\
\hline NIPEm & $62(56-71)$ & $61(57-66)$ & $1.075(1.012-1.141)^{c}$ & 0.02 \\
\hline NIPEi & $62(55-76)$ & $61(53-67)$ & $1.078(1.023-1.137)^{c}$ & 0.006 \\
\hline
\end{tabular}

OR calculated for deep analgosedation per (a) 1 week increase in PMA, (b) $100 \mathrm{~g}$ increase in weight, and (c) one unit increase in NIPE. 
W. Walas, E. Malinowska, Z.P. Halaba, T. Szczapa, J. Latka-Grot, M. Rutkowska, A. Kubiaczyk, M. Wrońska, A. Piotrowski, M. Skrzypek, M. Jean-Noel, I. Maroszyńska

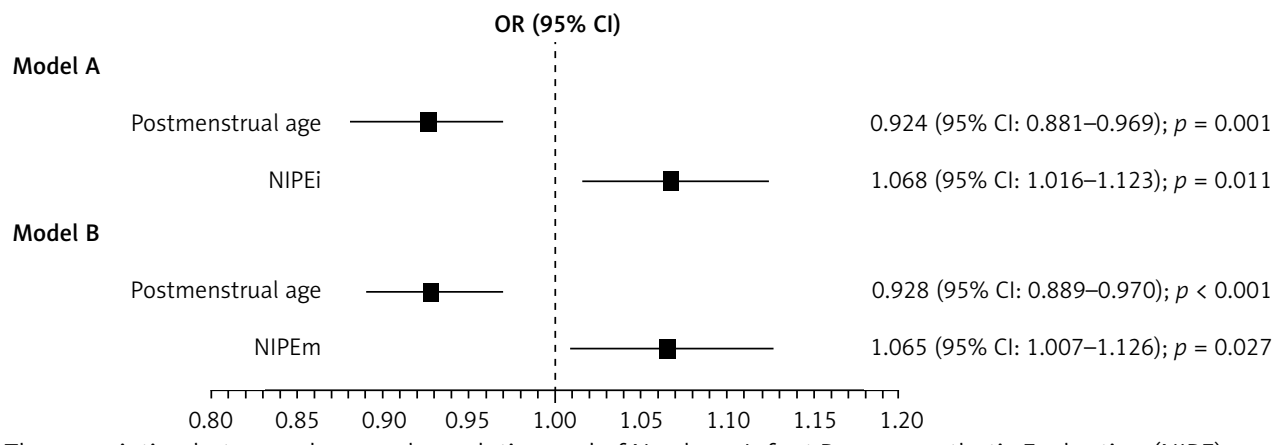

Figure 1. The association between deep analgosedation and of Newborn Infant Parasympathetic Evaluation (NIPE) indexes adjusted for confounders - results of multivariable logistic regression models with (A) NIPEi and (B) NIPEm included

OR calculated for deep analgosedation per 1 week increase in PMA and one unit increase in NIPE.

\section{Discussion}

Our study demonstrated that NIPE indexes are related to the levels of analgosedation. In the case of deep analgosedation the NIPE indexes are higher than in the case of moderate or insufficient analgosedation. Identification of deep analgosedation seems to be of particular practical importance in intubated and mechanically ventilated newborns and infants treated in P/NICUs. While the detection of insufficient analgosedation is usually easier due to the behavioral-physiological manifestation of pain, it can be difficult to distinguish between deep and too deep analgosedation. A COMFORT-B score $\leq 10$ is regarded in the literature as "deep sedation" or "oversedation" and no threshold has been established for drug overdose. In clinical practice, the line between deep and excessive analgosedation may be blurred. Furthermore, the assessment using the COMFORT-B score is periodical and a situation threatening the evolution of deep analgosedation to oversedation may be overlooked. Therefore, early identification of any situation where sedation is deep is essential. From this point of view, NIPE indexes may be helpful in identifying situations in which the patient may be at risk of oversedation.

The ability to detect deep analgosedation applies to both NIPE indexes, i.e. NIPEi and NIPEm. This is understandable taking into consideration the fact that during the study the patients received continuous infusions of the hypnotic drug and opioid and were not disturbed by additional stimuli during the measurements.

Most studies on the usefulness of HRV analysis in detecting and monitoring pain in newborns and infants address procedural pain. Lindh et al. found that in term and preterm neonates HRV analysis detects pain and enables evaluation of the effectiveness of EMLA (Eutectic Mixture of Local Anaesthetics) cream and oral glucose [28-31]. The usefulness of HRV analysis in detecting procedural pain in newborns and infants was also confirmed by Oberlander et al. [32, 33]. Weissman et al. as well as Cong et al. noted the usefulness of HRV analysis in assessing the usefulness of non-pharmacological methods of alleviating procedural pain in neonates $[34,35]$. The meta-analysis by Zhang et al. showed that HRV analysis was useful in studies of the effect of mother's milk odor on alleviating procedural pain in newborns [36]. In contrast, Cremillieux et al. obtained opposite results. They did not find any correlation between NIPE monitor measurements and assessments based on behavioral scales and came to the conclusion that the NIPE index is not a reliable tool for evaluation of acute pain in preterm infants [37]. From observations made by Walas et al. it follows that the time points selected by Cremillieux et al. may not be optimal to objectively assess the NIPE monitor [38].

While the usefulness of HRV analysis in detecting procedural pain in newborns and infants is beyond doubt, there are only very few papers on the application of this method in the assessment of analgosedation in patients from this age group treated in P/NICUs. Studies in adult patients show that HRV analysis may be useful in the assessment of pain in the postoperative period and in the intensive care unit [39-42]. However, we found only a few papers relating to newborns and infants. The authors' conclusions are similar to ours.

Research performed by Faye et al. in 28 newborns with gestational age > 34 weeks at 2-72 $\mathrm{h}$ after major surgical procedures concentrated on pain intensity assessed by means of the Échelle Douleur Inconfort Nouveau-Né (EDIN) scale and the high frequency variability index (HFVI) measured simultaneously. They noted that HFVI was significantly lower in the group of newborns with an EDIN score $\geq 5$ compared to newborns with an EDIN score $<5$ and concluded that HRV may be used as an indicator to assess prolonged pain in newborn infants [26]. Buyuktiryaki et al. found a correlation between the NIPE index and the EDIN score after insertion of a chest tube in 
newborns of gestational age between 33 and 35 weeks. They concluded that prolonged pain can be accurately assessed using NIPE index [43]. Gall et al. evaluated postoperative pain in children, including infants. They concluded that the HRV analysis may provide an objective measurement of acute postoperative pain, which is correlated with that measured with the FLACC (Face, Legs, Activity, Cry, and Consolability) scale [44]. Infants older than 6 months of age were included in the group of children undergoing muscle biopsy under analgesia and light sedation examined by Avez-Couturier et al. The pain was assessed using the FLACC scale and the HRV was analyzed simultaneously. The authors noted that the HRV decreased in response to the pain stimulus and that HRV and FLACC values changed by a similar magnitude in children both younger and older than 6 years [45]. Recher et al. performed research on newborns, infants and children $<3$ years of age treated in the PICU, who were intubated and under analgosedation, in which they compared changes in the NIPE index and the COMFORT score during various care procedures. They concluded that NIPEi is valid for assessing distress in sedated/intubated children in the PICU [46].

Our study has some limitations. The study group was small and diverse; therefore, we treat this study as a pilot study. The small number of cases in which the COMFORT score was $>10$ made it impossible to distinguish situations in which analgosedation was moderate (COMFORT-B: 11-23) vs. insufficient (COMFORT-B: > 23). Continued research is necessary with larger and less diverse patient populations.

In conclusion, the results of our research indicate that NIPE indexes are significantly higher in patients whose assessment on the behavioral scale indicates deep analgosedation as compared to those in whom it indicates moderate or insufficient analgosedation. Allowing continuous monitoring, the NIPE device may be a valuable assisting tool in the assessment of analgosedation quality in mechanically ventilated newborns and infants. Further studies are needed to confirm the usefulness of this method in clinical practice. Especially the determination of measurement thresholds corresponding to different pain intensities would be very useful.

\section{Conflict of interest}

The authors declare no conflict of interest.

\section{References}

1. Duerden EG, Grunau RE, Guo T, et al. Early procedural pain is associated with regionally-specific alterations in thalamic development in preterm neonates. J Neurosci 2018; 38: 878-86.
2. Schneider J, Duerden EG, Guo T, et al. Procedural pain and oral glucose in preterm neonates: brain development and sex-specific effects. Pain 2018; 159: 515-25.

3. Smith GC, Gutovich J, Smyser C, et al. Neonatal intensive care unit stress is associated with brain development in preterm infants. Ann Neurol 2011; 70: 541-9.

4. Brummelte S, Grunau RE, Chau V, et al. Procedural pain and brain development in premature newborns. Ann Neurol 2012; 71: 385-96.

5. Ranger M, Chau CM, Garg A, et al. Neonatal pain-related stress predicts cortical thickness at age 7 years in children born very preterm. PLoS One 2013; 8: e76702.

6. Brummelte S, Chau CM, Cepeda IL, et al. Cortisol levels in former preterm children at school age are predicted by neonatal procedural pain-related stress. Psychoneuroendocrinology 2015; 51: 151-63.

7. Hohmeister J, Kroll A, Wollgarten-Hadamek I, et al. Cerebral processing of pain in school-aged children with neonatal nociceptive input: an exploratory fMRI study. Pain 2010; 150: 257-67.

8. Abdulkader HM, Freer Y, Garry EM, Fleetwood-Walker SM, McIntosh N. Prematurity and neonatal noxious events exert lasting effects on infant pain behaviour. Early Hum Dev 2008; 84: 51-5.

9. Hermann C, Hohmeister J, Demirakça S, Zohsel K, Flor H. Long-term alteration of pain sensitivity in school-aged children with early pain experiences. Pain 2006; 125: 78-85.

10. Grunau RV, Whitfield MF, Petrie JH, Fryer EL. Early pain experience, child and family factors, as precursors of somatization: a prospective study of extremely premature and fullterm children. Pain 1994; 56: 353-9.

11. Simons SH, van Dijk M, van Lingen RA, et al. Routine morphine infusion in preterm newborns who received ventilatory support: a randomized controlled trial. JAMA 2003; 290: 2419-27.

12. Anand KJ, Hall RW, Desai N, et al. Effects of morphine analgesia in ventilated preterm neonates: primary outcomes from the NEOPAIN randomised trial. Lancet 2004; 363: 1673-82.

13. Bellù R, de Waal KA, Zanini R. Opioids for neonates receiving mechanical ventilation. Cochrane Database Syst Rev 2008; 1: CD004212.

14. Chen M, Shi X, Chen Y, et al. A prospective study of pain experience in a neonatal intensive care unit of China. Clin J Pain 2012; 28: 700-4.

15. Avila-Alvarez A, Carbajal R, Courtois E, Pertega-Diaz S, Anand KJ, Muńiz-Garcia J; Grupo español del proyecto Europain. Clinical assessment of pain in Spanish neonatal intensive care units. An Pediatr 2016; 85: 181-8 [Spanish].

16. Courtois E, Droutman S, Magny JF, et al. Epidemiology and neonatal pain management of heelsticks in intensive care units: EPIPPAIN 2, a prospective observational study. Int I Nurs Stud 2016; 59: 79-88.

17. Sposito NPB, Rossato LM, Bueno M, Kimura AF, Costa T, Guedes DMB. Assessment and management of pain in newborns hospitalized in a neonatal intensive care unit: a cross-sectional study. Rev Lat Am Enfermagem 2017; 25: e2931.

18. Lago P, Frigo AC, Baraldi E, et al. Sedation and analgesia practices at Italian neonatal intensive care units: results from the EUROPAIN study. Ital J Pediatr 2017; 43: 26.

19. Anand KJS, Eriksson M, Boyle EM, et al. Assessment of continuous pain in newborns admitted to NICUs in 18 European countries. Acta Paediatr 2017; 106: 1248-59.

20. Carbajal R, Rousset A, Danan C, et al. Epidemiology and treatment of painful procedures in neonates in intensive care units. JAMA 2008; 300: 60-70. 
21. Srouji R, Ratnapalan S, Schneeweiss S. Pain in children: assessment and nonpharmacological management. Int J Pediatr 2010; 2010: 474838

22. Beltramini A, Milojevic K, Pateron D. Pain assessment in newborns, infants, and children. Pediatr Ann 2017; 46 e387-95.

23. Kotfis K, Zegan-Barańska M, Strzelbicka M, Safranow K Żukowski M, Ely EW; POL-CPOT Study Group. Validation of the Polish version of the Critical Care Pain Observation Tool (CPOT) to assess pain intensity in adult, intubated intensive care unit patients: the POL-CPOT study. Arch Med Sci 2018; 14: 880-9.

24. Ista E, van Dijk M, Tibboel D, de Hoog M. Assessment of sedation levels in pediatric intensive care patients can be improved by using the COMFORT "behavior" scale. Pediatr Crit Care Med 2005; 6: 58-63.

25. Jeanne M, Logier R, De Jonckheere J, Tavernier B. Validation of a graphic measurement of heart rate variability to assess analgesia/nociception balance during general anesthesia. Annu Int Conf IEEE Eng Med Biol Soc 2009; 2009: 1840-3.

26. Faye PM, De Jonckheere J, Logier R, Jeanne M, Rakza T, Storme L. Newborn infant pain assessment using heart rate variability analysis. Clin J Pain 2010; 26: 777-82.

27. De Jonckheere J, Rakza T, Logier R, Jeanne M, Jounwaz R, Storme $L$. Heart rate variability analysis for newborn infants prolonged pain assessment. Annu Int Conf IEEE Eng Med Biol Soc 2011; 2011: 7747-50.

28. Lindh V, Wiklund U, Sandman PO, Håkansson S. Assessment of acute pain in preterm infants by evaluation of facial expression and frequency domain analysis of heart rate variability. Early Hum Dev 1997; 48: 131-42.

29. Lindh V, Wiklund U, Håkansson S. Heel lancing in term new-born infants: an evaluation of pain by frequency domain analysis of heart rate variability. Pain 1999; 80: 143-8.

30. Lindh V, Wiklund U, Híkansson S. Assessment of the effect of EMLA during venipuncture in the newborn by analysis of heart rate variability. Pain 2000; 86: 247-54.

31. Lindh V, Wiklund U, Blomquist HK, Håkansson S. EMLA cream and oral glucose for immunization pain in 3-month-old infants. Pain 2003; 104: 381-8.

32. Oberlander TF, Grunau RE, Pitfield S, Whitfield MF, Saul JP. The developmental character of cardiac autonomic responses to an acute noxious event in 4- and 8-monthold healthy infants. Pediatr Res 1999; 45: 519-25.

33. Oberlander TF, Grunau RE, Whitfield MF, Fitzgerald C, Pitfield S, Saul JP. Biobehavioral pain responses in former extremely low birth weight infants at four months' corrected age. Pediatrics 2000; 105: e6.

34. Weissman A, Aranovitch M, Blazer S, Zimmer EZ. Heellancing in newborns: behavioral and spectral analysis assessment of pain control methods. Pediatrics 2009; 124: e921-6.

35. Cong X, Ludington-Hoe SM, McCain G, Fu P. Kangaroo care modifies preterm infant heart rate variability in response to heel stick pain: pilot study. Early Hum Dev 2009; 85: 561-7.

36. Zhang S, Su F, Li J, et al. The analgesic effects of maternal milk odor on newborns: a meta-analysis. Breastfeed Med 2018; 13: 327-34.

37. Cremillieux C, Makhlouf A, Pichot V, Trombert B, Patural $\mathrm{H}$. Objective assessment of induced acute pain in neonatology with the Newborn Infant Parasympathetic Evaluation index. Eur J Pain 2018; 22: 1071-9.

38. Walas W, Latka-Grot J, Maroszyńska I, et al. Newborn infant parasympathetic evaluation index for the assess- ment of procedural pain in nonanesthetized infants: a multicenter pilot study. Am J Perinatol 2020; doi: 10.1055/s-0040-1709458.

39. Chanques G, Tarri T, Ride A, et al. Analgesia nociception index for the assessment of pain in critically ill patients: a diagnostic accuracy study. Br J Anaesth 2017; 119: 812-20.

40. Jendoubi A, Abbes A, Ghedira S, Houissa M. Pain measurement in mechanically ventilated patients with traumatic brain injury: behavioral pain tools versus analgesia nociception index. Indian J Crit Care Med 2017; 21 : 585-8.

41. Papaioannou V, Chouvarda I, Gaertner E, et al. Heart rate variability and cardiac baroreflex inhibition-derived index predicts pain perception in burn patients. Burns 2016; 42: 1445-54.

42. Broucqsault-Dédrie C, De Jonckheere J, Jeanne M, Nseir S. Measurement of heart rate variability to assess pain in sedated critically ill patients: a prospective observational study. PLoS One 2016; 11: e0147720.

43. Buyuktiryaki $M$, Uras $N$, Okur $N$, et al. Evaluation of prolonged pain in preterm infants with pneumothorax using heart rate variability analysis and EDIN (Échelle Douleur Inconfort Nouveau-Né, neonatal pain and discomfort scale) scores. Korean J Pediatr 2018; 61: 322-6.

44. Gall O, Champigneulle B, Schweitzer B, et al. Postoperative pain assessment in children: a pilot study of the usefulness of the analgesia nociception index. Br J Anaesth 2015; 115: 890-5.

45. Avez-Couturier J, De Jonckheere J, Jeanne M, Vallée L, Cuisset JM, Logier R. Assessment of procedural pain in children using analgesia nociception index: a pilot study. Clin J Pain 2016; 32: 1100-4.

46. Recher M, Rousseaux J, Flocteil M, et al. Assessment of procedural distress in sedated/intubated children under 3 years old using the newborn infant parasympathetic evaluation: a diagnostic accuracy pilot study. Pediatr Crit Care Med 2020; 21: e1052-60. 